\title{
Running Test on Current Collector with Contact Force Controller for High-Speed Railways*
}

\author{
Toshiaki MAKINO**, Kazuo YOSHIDA***, \\ Shinji SETO**** and Kazuhiro MAKINO**
}

\begin{abstract}
To reduce noise caused by current collectors on high-speed railways, a wingshaped low-noise current collector has been developed. This collector head is directly supported by a composite insulator. However, it becomes difficult to maintain the predetermined contact force of the contact strip against the trolley wire. Therefore, the vertical height of those apparatus should be controlled actively. The upper part of the composite insulator is under high voltage. When using sensors only under the insulator, it is difficult to distinguish wire disturbance from lift disturbance. In this study, the difficulty is solved by using $H_{\infty}$ controller to estimate those disturbances in each frequency range. In traveling experiments using full-size prototype equipment on the running vehicle, it was confirmed that the contact force variation due to lift of 68 $\mathrm{N}$ at the speed of $112 \mathrm{~km} / \mathrm{h}$ was reduced by about $33 \%$ compared with active control without lift compensation, and the wire push-up variation was reduced by about $67 \%$.
\end{abstract}

Key Words : Contact Force Control, $H_{\infty}$ Control Method, Hydraulic Mechanism Current Collector, High-Speed Railways

\section{Introduction}

Throughout the world, high-speed transportation has become increasingly prevalent in recent years. This is exemplified by France's TGV, Germany's ICE and Japan's Shinkansen ("Bullet") trains. Bullet trains are driven at high speed through densely populated residential areas. Thus, suppressing noise and vibration to the greatest possible extent is a matter of considerable importance $e^{(1),(2)}$. In particular, for the latest Shinkansen trains which run at high speeds of approximately $350 \mathrm{~km} / \mathrm{h}$, it is essential to reduce aerodynamic noise from current collectors that cannot be expected to be reduced by building noise barriers.

One method that has been used to reduce current

* Received 9th December, 1996

** Hitachi Ltd., Kasado Works, 794 Higashitoyoi, Kudamatsu-shi, Yamaguchi 744, Japan

*** Faculty of Science and Technology, Keio University, 3-14-1 Hiyoshi, Kouhoku-ku, Yokohama-shi, Kanagawa 223, Japan

**** Graduate School of Science and Technology, Keio University collector noise involves surrounding the current collector with a pantograph cover having a cavity ${ }^{(3)}$. However, the pantograph cover itself is also a source of aerodynamic noise. The transmission, into the train, of hydrodynamic vibration resulting from the separation of the air stream around the pantograph cover is also starting to cause problems ${ }^{(4),(5)}$.

Therefore, a new concept of changing the configuration of the low-noise current collector to decrease the noise has been developed ${ }^{(6)}$. This current collector principally consists of a wing-shaped current collector, a supporting insulator, and a stream lined dome. However, it was found that the main stream hits the head of the modified low-noise current collector configuration. As a result, the lift acting on the collector head was increased to about ten times as much as the conventional level, and the contact force acting between the trolley wire and the contact strip exceeded its allowance ${ }^{(7)}$. In order to maintain the performance of the current collector at the conventional level, it is considered that active control on the contact force control system is indispensable. Methods for actively controlling the current collector using a contact force control system have already 
been proposed ${ }^{(8)-(11)}$. However, they require the installation of many sensors on the high-voltage part of the contact strip. With conventional technology, there are problems in that multiple sensors cannot be installed on the high-voltage part, and that it is impossible to detect the contact force directly.

We report a method of active control using a contact force control system ${ }^{(12)}$. We also investigate a contact force control method based on $H_{\infty}$ control, wherein, unlike conventional methods, the contact force is estimated from signals obtained from multiple sensors provided on ground voltage parts. Furthermore, we conducted test runs of full-size prototype equipment on a running vehicle at the Railway Technical Research Institute (R. T. R. I.), and evaluated the trolley wire tracking performance. As a result, it was confirmed that the contact force variation with a lift of $68 \mathrm{~N}$ at a traveling speed of $112 \mathrm{~km} / \mathrm{h}$ is reduced by about $67 \%(30 \mathrm{~N})$, and the wire push-up variation is reduced by about $33 \%$.

\section{Configuration of Low-Noise Current Collector}

\subsection{Configuration}

Figures 1(a) and (b) show the configuration of the low-noise current collector ${ }^{(12)}$. This collector includes a streamlined dome mounted on the train roof, a contact strip that touches the trolley wire and collects electric power, a delta-wing incorporating a leaf spring mechanism that supports the contact strip at two points, and a supporting insulator that provides insulation and supports the leaf spring mechanism. Although not illustrated, the contact strip moves along its vertical direction with a maximum stroke of

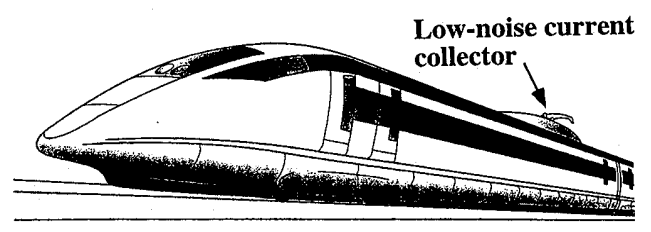

(a) Low-noise current collector on the high-speed train

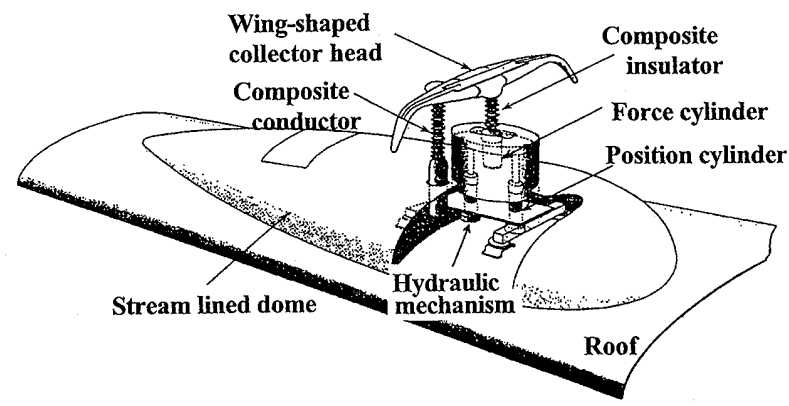

(b) Configuration of the low-noise current collector

Fig. 1 Conceptual configuration of the low-noise current collector
$17 \mathrm{~mm}$, and can track the trolley wire with a wirehanger (about $5 \mathrm{~m}$ ) displacement at high frequencies ( $10 \mathrm{~Hz}$ and above). Also, the wing-shaped collector head on which the lift acts (the contact strip and delta- wing collector head) is directly supported and insulated by the composite insulator. The parts below the composite insulator are thereby at ground voltage, and thus multiple detectors can be installed. With this configuration, the nonobservable force of interaction between the trolley wire and the contact strip is indirectly estimated internally. The two-step hydraulic mechanism comprises a force cylinder in the upper part and a position cylinder in the lower part. These are driven by control signals (mentioned below) based on the above-mentioned detection signal outputs. The force cylinder and the position cylinder are controlled independently of each other. The stroke of the force cylinder is $100 \mathrm{~mm}$, and the stroke of the position cylinder is $600 \mathrm{~mm}$.

\subsection{Analytical model of trolley wire/current collector system}

Figure 2 shows a schematic of the analysis model of the trolley wire/current collector system ${ }^{(13)}$. The trolley wire and the contact strip are assumed to be in constant contact; the case of separation from the trolley wire is not considered. The spring and damping coefficients of the trolley wire $\left(k_{w}, c_{w}\right)$ are assumed to be constant, and effects such as of the vertical and lateral vibration of the train and longitudinal vibration of the wing-shaped current collector are not considered. A passive model is obtained by substituting the force cylinder with a stiff spring and damper $\left(k_{w}=3.92 \times 10^{6} \mathrm{~N} / \mathrm{m}, c_{w}=1.61 \times 10^{4} \mathrm{Ns} / \mathrm{m}\right) . z_{w}(t)$

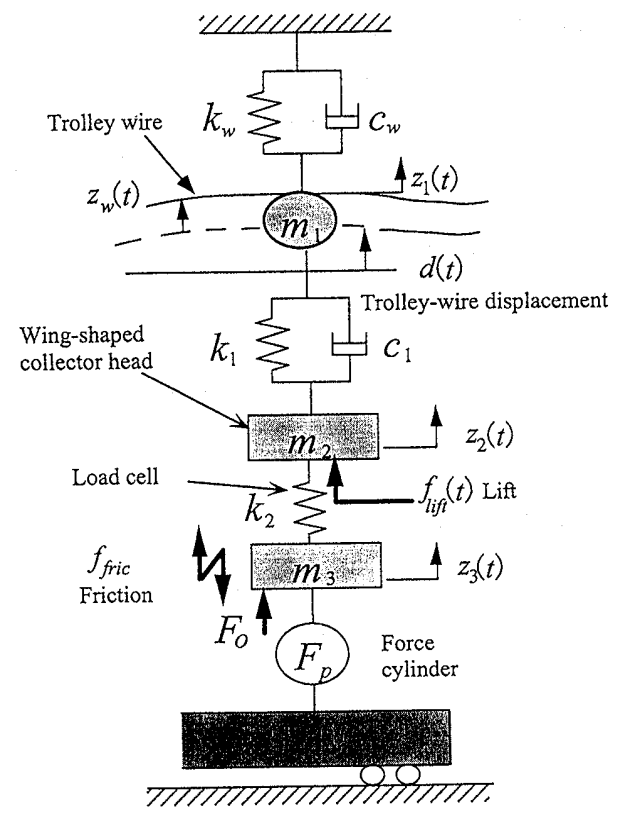

Fig. 2 Schematics of analysis model of the trolley wire/current collector system 
is trolley wire displacement.

The equations of motion for the trolley wire/ current collector system model are

$$
\begin{aligned}
& m_{1} \ddot{z}_{1}(t)=-k_{1}\left(z_{1}(t)-z_{2}(t)\right)-c_{1}\left(\dot{z}_{1}(t)\right. \\
& \left.\quad-\dot{z}_{2}(t)\right)-f(t) \\
& m_{2} \ddot{z}_{2}(t)=k_{1}\left(z_{1}(t)-z_{2}(t)\right)-c_{1}\left(\dot{z}_{1}(t)\right. \\
& \left.\quad-\dot{z}_{2}(t)\right)-k_{2}\left(z_{2}(t)-z_{3}(t)\right)+f_{\text {lift }}(t) \\
& m_{3} \ddot{z}_{3}(t)=k_{2}\left(z_{2}(t)-z_{3}(t)\right)+F_{p}(t)+f_{\text {fric }}(t) \\
& f(t)=k_{w} z_{w}(t)+c_{w} \dot{z}_{w}(t) \\
& z_{1}(t)=z_{w}(t)+d(t)
\end{aligned}
$$

The equations of motion in Eqs. (1) through (5) can be represented as the following state equations.

$$
\begin{aligned}
& \dot{\boldsymbol{x}}_{s}(t)=\boldsymbol{A}_{s} \boldsymbol{x}_{s}(t)+\boldsymbol{B}_{s u} F_{p}(t)+\boldsymbol{B}_{s d} \boldsymbol{d}(t) \\
& +\boldsymbol{B}_{s l} f_{\text {lift }}(t)+\boldsymbol{B}_{\text {sf }} f_{\text {fric }}(t) \\
& \boldsymbol{x}_{s}(t)=\left[\begin{array}{llllll}
z_{1}(t) & \dot{z}_{1}(t) & z_{2}(t) & \dot{z}_{2}(t) & z_{3}(t) & \dot{z}_{3}(t)
\end{array}\right]^{T} \\
& \boldsymbol{d}(t)=\left[\begin{array}{ll}
d(t) & \dot{d}(t)
\end{array}\right]^{T}
\end{aligned}
$$

Figure 3 shows the block diagram of hydraulic force control system, which consists of a disturbance observer and a desired value filter. This control system compensates the hydraulic system from the effects of the input signal $(u(t))$ of the servo amplifier to the control force $\left(F_{p}(t)\right)$. Figure 4 shows the frequency response of hydraulic force control system $\left(F_{p}(t) / F_{p r}(t)\right)$, according to calculated results and measured results. From these results, the hydraulic force control system is considered to be the secondorder system described by Eqs. ( 7), ( 8 ) and (9). By

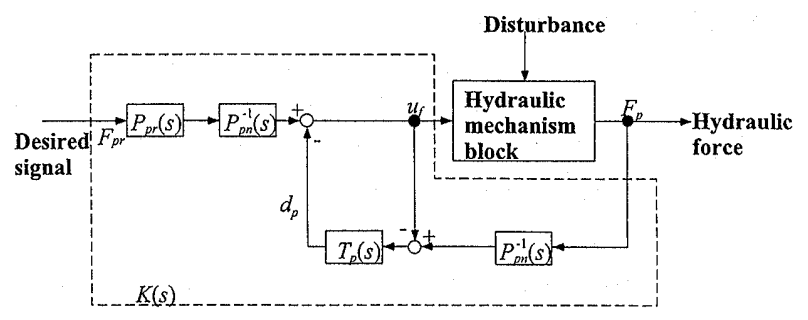

Fig. 3 Block diagram of the hydraulic force control system using a disturbance observer
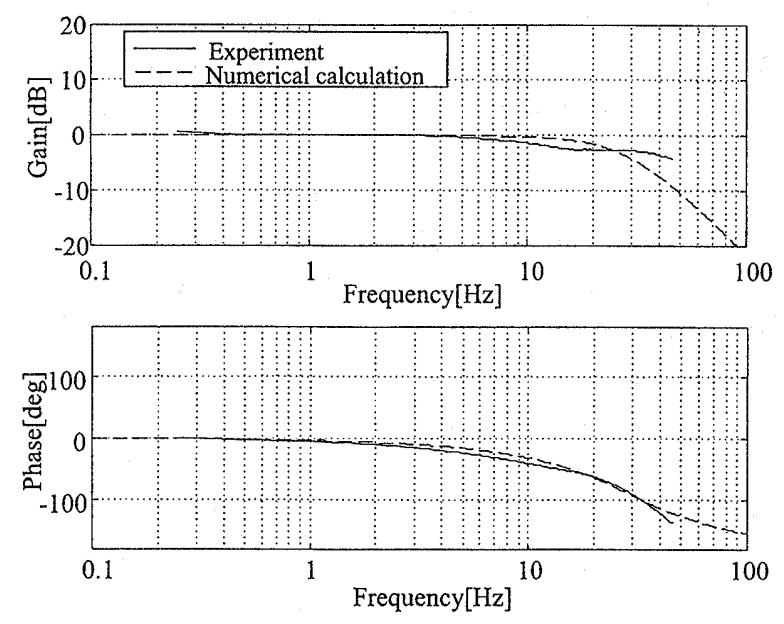

Fig. 4 Frequency responses of the hydraulic force control system $\left(F_{p} / F_{p r}\right)$ using this control system, the control force $\left(F_{p}(t)\right)$ is applied to the force cylinder (single-rod type) through a hydraulic servo valve (flow control valve), and the effect of hydraulic force characteristics can be eliminated by the difference in surface areas. It is also possible to compensate the nonlinear characteristics and system parameter variations arising from frictional forces in the linear bearings that support the hydraulic servo valve and the force cylinder ${ }^{(14)}$.

$$
\begin{aligned}
& \frac{F_{p}(s)}{F_{p r}(s)}=\frac{\omega_{a c}^{2}}{s^{2}+2 \zeta_{a c} \omega_{a c} s+\omega_{a c}^{2}} \\
& \dot{\boldsymbol{x}}_{a c}(t)=\boldsymbol{A}_{a c} \boldsymbol{x}_{a c}(t)+\boldsymbol{B}_{a c} F_{p r}(t) \\
& F_{p}(t)=\boldsymbol{C}_{a c} \boldsymbol{x}_{a c}(t)
\end{aligned}
$$

Next, a model of the disturbance system is postulated. The trolley wire has periodic irregularities due to distance $(50 \mathrm{~m})$ between points where hanging wires are supported by poles and the intervals between points of hangers supporting the trolley wire $(5 \mathrm{~m})$. These trolley wire irregularities can be represented as a power spectral density having two peaks that vary with speed ${ }^{(8)}$. Thus, the trolley wire disturbance has peaked dynamics, as shown in Fig. 5. Its transfer function and state equation are shown by Eqs. (10), (11) and (12). Here, $w_{d}(t)$ is white noise.

$$
\begin{aligned}
& \frac{d(s)}{w_{d}(s)}=\frac{k_{d} \omega_{d 1}^{2} s}{\left(s^{2}+2 \zeta_{d 1} \omega_{d 1} s+\omega_{d 1}^{2}\right)\left(s^{2}+2 \zeta_{d 2} \omega_{d 2} s+\omega_{d 2}^{2}\right)} \\
& \dot{\boldsymbol{x}}_{d}(t)=\boldsymbol{A}_{d} \boldsymbol{x}_{d}(t)+\boldsymbol{B}_{d} w_{d}(t) \\
& \boldsymbol{d}(t)=\boldsymbol{C}_{d} \boldsymbol{x}_{d}(t)
\end{aligned}
$$

The frictional force in the force cylinder $(30 \mathrm{~N})$ is of the same strength as the contact force in the lowfrequency range. To compensate this frictional force, assuming a stepped disturbance is applied, the transfer function and state equation are shown in Eqs. (13) and (14). Here, $w_{\text {fric }}(t)$ is white noise.

$$
\begin{aligned}
& f_{\text {fric }}(s) / w_{\text {fric }}(s)=c_{\text {fric }} / s \\
& \dot{f}_{\text {fric }}(t)=c_{\text {fric }} w_{\text {fric }}(t)
\end{aligned}
$$

The lift acting on the wing-shaped collector head consists of steady lift due to the traveling speed, and

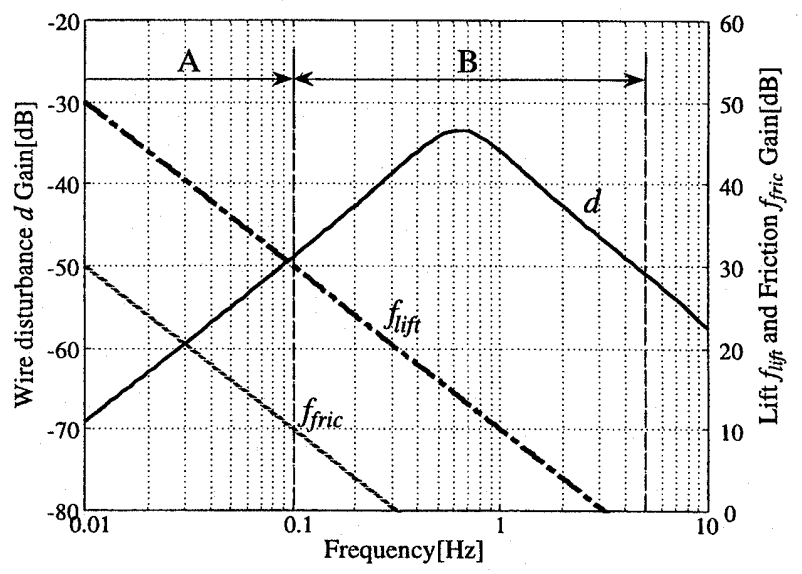

Fig. 5 Frequency responses of disturbance models 
variable lift due to pressure variations that occur when entering and exiting tunnels. Therefore, assuming that the effect of on the steady value of the contact force is large, this transfer function and state equation are shown in Eqs. (15) and (16). Here, $w_{\text {lift }}(t)$ is white noise.

$$
\begin{aligned}
& f_{\text {lift }}(s) / w_{\text {lift }}(s)=c_{\text {lift }} / s \\
& \dot{f}_{\text {lift }}(t)=c_{\text {lift }} w_{\text {lift }}(t)
\end{aligned}
$$

As shown in Fig. 5, the disturbances are divided, according to frequency, into zone $\mathrm{A}(0.1 \mathrm{~Hz}$ or less) and zone $\mathrm{B}$ (from $0.1 \mathrm{~Hz}$ to $6 \mathrm{~Hz}$ ). The state equation of this trolley wire/current collector system is expressed by the expanded system state equation (17), which includes the external disturbance dynamics of the trolley wire irregularities, lift and so on, and a hydraulic force control system dynamics.

$$
\begin{aligned}
& \dot{\boldsymbol{x}}_{p}(t)=\boldsymbol{A}_{p} \boldsymbol{x}_{p}(t)+\boldsymbol{B}_{p e} F_{p r}(t)+\boldsymbol{B}_{p w} \boldsymbol{w}_{p}(t) \\
& \boldsymbol{x}_{p}(t)=\left[\begin{array}{lllll}
\boldsymbol{x}_{s}^{T}(t) & \boldsymbol{x}_{d}^{T}(t) & \boldsymbol{x}_{a c}^{T}(t) & f_{\text {lift }}(t) & f_{\text {fric }}(t)
\end{array}\right] \\
& \boldsymbol{w}_{p}(t)=\left[\begin{array}{lll}
w_{d}(t) & w_{\text {lift }}(t) & w_{\text {fric }}(t)
\end{array}\right]^{T}
\end{aligned}
$$

The detected quantities are taken as the force $\left(f_{z}(t)\right)$ of the load cell, the upward acceleration $\left(\ddot{z}_{2}(t)\right)$ of the load cell, and the displacement $\left(z_{3}(t)\right)$ and acceleration $\left(\ddot{z}_{3}(t)\right)$ of the force cylinder. These detected values and the controlled value of contact force $(f(t))$ are expressed by

$$
\begin{aligned}
& \boldsymbol{y}(t)=\left[\begin{array}{llll}
f_{z}(t) & z_{3}(t) & \ddot{z}_{2}(t) & \ddot{z}_{3}(t)
\end{array}\right]^{T} \\
& =\boldsymbol{C}_{s} \boldsymbol{x}_{s}(t)+\boldsymbol{D}_{s i} f_{\text {lift }}(t)+\boldsymbol{D}_{s f} f_{\text {fric }}(t)+\boldsymbol{D}_{s u} F_{p}(t) \\
& =\boldsymbol{C}_{p} \boldsymbol{x}_{p}(t)+\boldsymbol{D}_{p v} \boldsymbol{v}(t) \\
& \boldsymbol{C}_{s}=\left[\begin{array}{cccccc}
0 & 0 & k_{2} & 0 & -k_{2} & 0 \\
0 & 0 & 0 & 0 & 1 & 0 \\
\frac{k_{1}}{m_{2}} & \frac{c_{1}}{m_{2}} & -\frac{k_{1}+k_{2}}{m_{2}} & -\frac{c_{1}+c_{2}}{m_{2}} & \frac{k_{2}}{m_{2}} & \frac{c_{2}}{m_{2}} \\
0 & 0 & \frac{k_{2}}{m_{3}} & \frac{c_{2}}{m_{3}} & -\frac{k_{2}}{m_{3}} & -\frac{c_{2}}{m_{3}}
\end{array}\right] \\
& \boldsymbol{D}_{s l}=\left[\begin{array}{c}
0 \\
0 \\
\frac{1}{m_{2}} \\
0
\end{array}\right], \boldsymbol{D}_{s f}=\left[\begin{array}{c}
0 \\
0 \\
0 \\
\frac{1}{m_{3}}
\end{array}\right], \boldsymbol{D}_{s u}=\left[\begin{array}{c}
0 \\
0 \\
0 \\
\frac{1}{m_{3}}
\end{array}\right] \\
& \boldsymbol{C}_{p}=\left[\begin{array}{lllll}
\boldsymbol{C}_{s} & \boldsymbol{0}_{(1 \times 4)} & \boldsymbol{D}_{s u} \boldsymbol{C}_{s} & \boldsymbol{D}_{s l} & \boldsymbol{D}_{s f}
\end{array}\right] \\
& \boldsymbol{v}(t)=\left[\begin{array}{llll}
v_{1}(t) & v_{2}(t) & v_{3}(t) & v_{4}(t)
\end{array}\right]^{T} \\
& f(t)=\boldsymbol{C}_{s o} \boldsymbol{x}_{s}(t)+\boldsymbol{D}_{s o} \boldsymbol{d}(t)=\boldsymbol{C}_{p o} \boldsymbol{x}_{p}(t)
\end{aligned}
$$

\section{Contact Force Control System Using $H_{\infty}$ Control}

The variations in the contact force that act upon the trolley wire and the contact strips have been maintained at previous levels through the use of contact force control in our newly developed low-noise current collectors. In the low-frequency range of 0.1 $\mathrm{Hz}$ and less, the disturbance in stationary lift is slight $(210 \mathrm{~N}$ at $0.1 \mathrm{~Hz}$ and lower at $350 \mathrm{~km} / \mathrm{h})$. In the frequency range of 0.2 to $8.0 \mathrm{~Hz}$, it is made to follow the irregularities in the trolley wire (a vibration width of $25 \mathrm{~mm}$ under an applied vibration frequency of 1.94 $\mathrm{Hz}$ generated when the span interval of the trolley wire is $50 \mathrm{~mm}$ and the speed is $350 \mathrm{~km} / \mathrm{h}$ ). In the highfrequency range above $10.0 \mathrm{~Hz}$, the use of passive control that employs a support mechanism for the contact strips follows the displacements that are caused by applied vibration frequencies (a vibration width of $1.0 \mathrm{~mm}$ at $19.4 \mathrm{~Hz}$ ). When the interval between the trolley wire hangers is $5 \mathrm{~m}$, the applied vibration frequencies are due to unevenness in the undulations that result from wear $(0.1 \mathrm{~mm}$ at 30 to 40 $\mathrm{Hz}$ ).

Accordingly, the first two design specifications are performed by means of two types of contact force control. Therefore, a cut was made for frequencies above $8.0 \mathrm{~Hz}$ since it is not necessary to provide for control of these higher frequencies against the variations in contact force that originate from the span intervals between the support points of the trolley wire. The weighting function, at this time, was set to satisfy the design specification by a trial-and-error method. Here, the control quantity was the contact force $f(t)$. The observed quantity was the relative displacements of load cells installed at the grounded potential sections, of acceleration meters placed in the upper and lower parts of these sections, and of force cylinders.

However, as noted in Ref.(15), from these observed quantities alone, it is impossible to observe or control the simultaneous effects of the disturbances of the lift and the displacement of the trolley wire irregularities on the trolley wire and current collector system. In estimates made using Kalman filters, simultaneous estimates of the disturbances could not be simultaneously estimated since there was a trade-off relationship in which an improvement in one of those factors led to a drop in the performance of the other in the low-frequency range. For this reason, as mentioned herein, a method has been used by which the quantity of each type of disturbance could be estimated after the ranges of frequencies for disturbance were first divided into zone $\mathrm{A}$ (for lift) and zone $B$ (for overhead wire irregularities).

\section{1 Configuration of generalized plant}

The contact force control system was designed using the output feedback method ${ }^{(16)}$. This feedback control internally stabilizes a closed loop system using the control formula $\boldsymbol{F}_{p r}(t)=\boldsymbol{H}(s) \boldsymbol{y}(t)$. Then $\boldsymbol{H}(s)$ must be determined such that the closed loop transfer function $G(s)$ from the disturbance to the evaluation output $\boldsymbol{Z}(t)=\left[\begin{array}{ll}Z_{1}(t) & Z_{2}(t)\end{array}\right]^{T}=\left[W_{f}(s) f(t)\right.$ $\left.W_{u}(s) F_{p r}(t)\right]^{T}$ satisfies $\|G(s)\|_{\infty}<\gamma$ for $\gamma>0$. Here, $W_{f}(s)$ and $W_{u}(s)$ are frequency weighting functions. The controlled quantity $\left(Z_{1}(t)\right)$ is the product of the 
contact force $(f(t))$ and weighting function $W_{f}(s)$. The weighting function $W_{f}(s)$ is expressed by Eq. (20), and its frequency characteristics are shown in Fig. 6.

$$
W_{f}(s)=\frac{\omega_{f}^{2}}{s^{2}+2 \zeta_{f} \omega_{f} s+\omega_{f}^{2}} L_{f}
$$

Here, $\omega_{f}=8 \mathrm{~Hz}, \zeta_{f}=0.7, L_{f}=3$

The characteristics of weighting function $W_{f}(s)$ are taken as a second-order low-pass filter to suppress fluctuation of the contact force at low frequencies.

On the other hand, the controlled quantity $\left(Z_{2}(t)\right)$ is the product of the desired control force $\left(F_{p r}(t)\right)$ and the weighting function $W_{u}(s) . W_{u}(s)$ is expressed as Eq. (21), and its frequency characteristics are shown in Fig. 6.

$$
W_{u}(s)=\frac{k\left(s^{2}+2 \omega_{h h} \zeta_{h h} s+\omega_{h h}^{2}\right)\left(s^{2}+2 \omega_{l h} \zeta_{l h} s+w_{l h}^{2}\right)}{\left(\left(s^{2}+2 \omega_{h l} \zeta_{h l} s+\omega_{h l}^{2}\right)\left(s^{2}+2 s \omega_{l l} \zeta_{l l}+\omega_{l l}^{2}\right)\right)}
$$

Here,

$$
\begin{aligned}
& \omega_{h h}=400 \mathrm{~Hz}, \omega_{l h}=0.1 \mathrm{~Hz}, \zeta_{h h}=\zeta_{l h}=0.7, k=1 \\
& \omega_{h l}=8 \mathrm{~Hz}, \omega_{l l}=0.005 \mathrm{~Hz} \text { and } \zeta_{h l}=\zeta_{l l}=0.7
\end{aligned}
$$

The characteristics of weighting function $W_{u}(s)$ are taken as a forth-order notch-filter to suppress the control force $\left(F_{p r}(t)\right)$ at high frequencies.

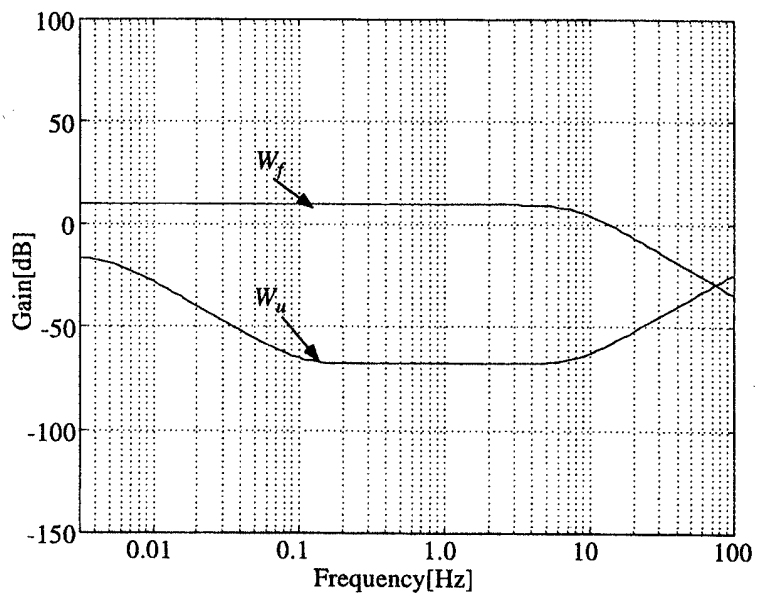

Fig. 6 Frequency characteristics of weighting functions

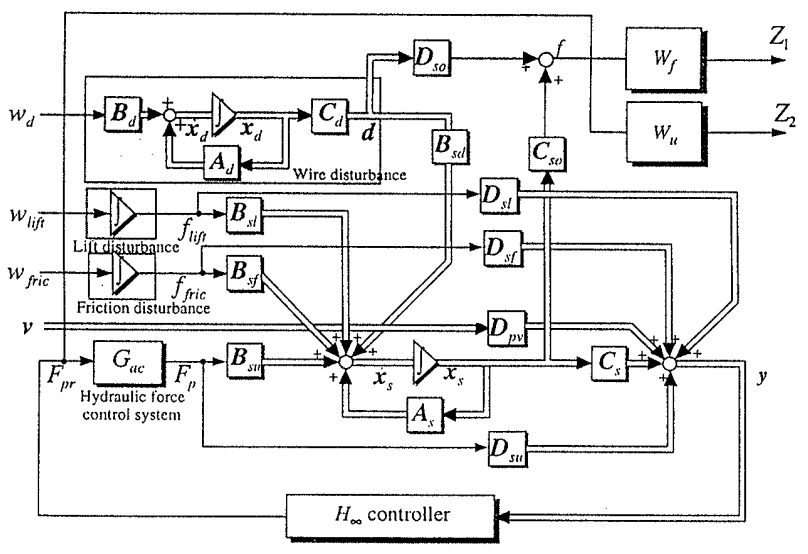

Fig. 7 Block diagram of generalized plant
Equations (22), (23) and (24) show formulas for the generalized plant of the contact force control system. A block diagram is shown in Fig. 7.

$$
\begin{aligned}
& \dot{\boldsymbol{x}}(t)=\boldsymbol{A} \boldsymbol{x}(t)+\boldsymbol{B}_{1} \boldsymbol{w}(t)+\boldsymbol{B}_{2} F_{p r}(t) \\
& \boldsymbol{Z}(t)=\boldsymbol{C}_{1} \boldsymbol{x}(t)+\boldsymbol{D}_{12} F_{p r}(t) \\
& \boldsymbol{y}(t)=\boldsymbol{C}_{2} \boldsymbol{x}(t)+\boldsymbol{D}_{21} \boldsymbol{w}(t) \\
& \boldsymbol{x}(t)=\left[\begin{array}{lll}
\boldsymbol{x}_{w f}^{T}(t) & \boldsymbol{x}_{w u}^{T}(t) & \boldsymbol{x}_{p}^{T}(t)
\end{array}\right]^{T} \\
& \boldsymbol{w}(t)=\left[\begin{array}{lll}
\boldsymbol{w}_{p}^{T}(t) & \boldsymbol{v}^{T}(t)
\end{array}\right]^{T}
\end{aligned}
$$

By solving the nonstandard problem from this standardized plant using Riccati's inequality, we derived the present $H_{\infty}$ controller $(H(s))^{(18),(19)}$.

$$
\begin{aligned}
& \dot{\hat{\boldsymbol{x}}}(t)=\widehat{\boldsymbol{A}} \widehat{\boldsymbol{x}}(t)+\widehat{\boldsymbol{B}} \boldsymbol{y}(t) \\
& F_{p r}(t)=\widehat{\boldsymbol{C}} \hat{\boldsymbol{x}}(t) \\
& \boldsymbol{H}(t)=\widehat{\boldsymbol{C}}(s \boldsymbol{I}-\widehat{\boldsymbol{A}})^{-1} \widehat{\boldsymbol{B}}
\end{aligned}
$$

This controller determines the gains of the feedback including the observers. Figure 8 shows the frequency characteristics obtained by estimating the displacement disturbance $(d(t))$ of the trolley wire irregularities and the disturbance $\left(f_{\text {lift }}(t)\right)$ of the lift in this system. It is impossible to separate the lift disturbance $\left(f_{\text {lift }}(t)\right)$ and the displacement disturbance $(d(t))$ simply from the quantities obtained from the detectors installed below the supporting insulator. However, as mentioned above, since the disturbance is separated into zone A and zone B frequency bands, the disturbance in each zone can be estimated. That is, as shown by the dotted line, at frequencies below $0.1 \mathrm{~Hz}$, the gain $\widehat{f}_{\text {lift }}(t) / f_{\text {lift }}(t)$ becomes $0 \mathrm{~dB}$, and the disturbance of steady lift can be estimated. Also, as shown by the solid line, in the frequency range from $0.2 \mathrm{~Hz}$ to $8 \mathrm{~Hz}$, the gain $\vec{d}(t) / d(t)$ becomes $0 \mathrm{~dB}$, and it is possible to estimate the displacement disturbance of the trolley wire irregularities. Transfer functions of $\widehat{d}(t) / d(t)$ and $\widehat{f}_{\text {lift }}(t) / f_{\text {lift }}(t)$ indicate frequency responses from $d(t)$ of the $W_{d}(t)$ system and $f_{\text {lift }}(t)$ of the $W_{\text {lift }}(t)$ system to $\hat{d}(t)$ and $\widehat{f}_{\text {lift }}(t)$ used in the $\overline{\boldsymbol{x}}(t)$ system.

\section{2 Simulation study}

Figures 9 shows the frequency characteristics of contact force $(f(t))$ with respect to the displacement disturbance $(d(t))$ of the trolley wire irregularity. In the frequency range from 0.2 to $6 \mathrm{~Hz}$, incontrast to the passive case shown by the dotted line, the active case shown by the solid line has lower gain and a better disturbance suppression effect. For example, in the

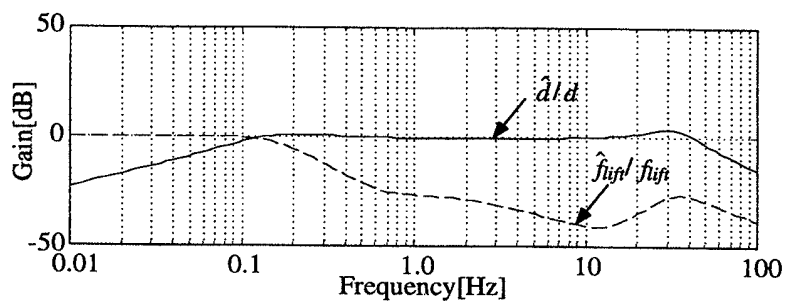

Fig. 8 Frequency responses of disturbance estimation 


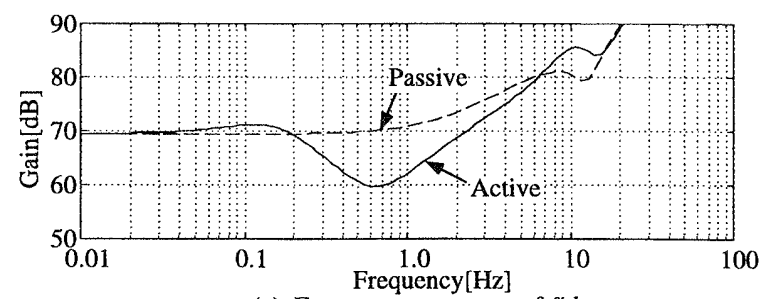

(a) Frequency responses of $f / d$

Fig. 9 Calculated responses of contact force and control force with a wire disturbance
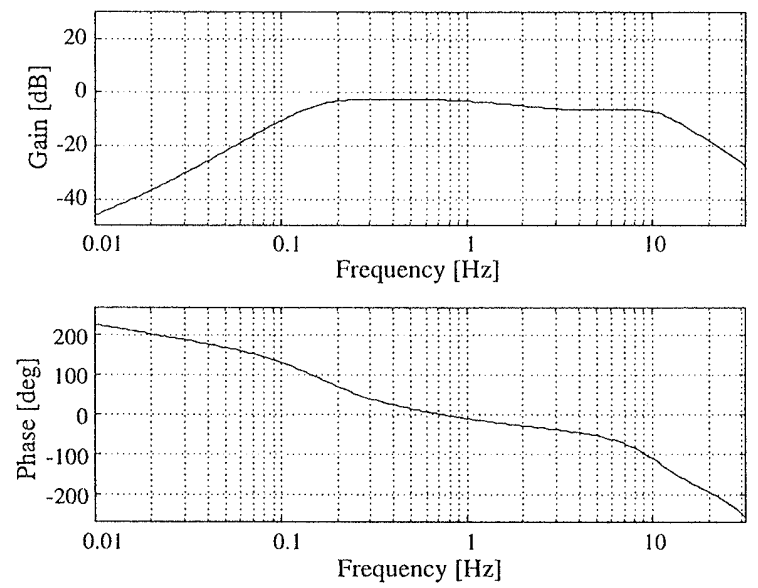

(a) Frequency response of contact force due to a lift disturbance
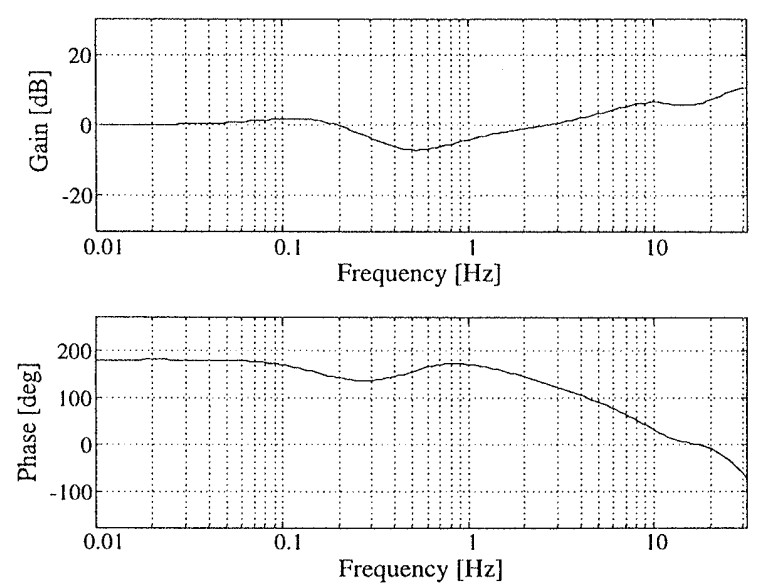

(b) Frequency response of control force due to a lift disturbance

Fig. 10 Calculated responses of contact force and control force with a lift disturbance

range from 1 to $2 \mathrm{~Hz}$, the gain is +5 to $8 \mathrm{~dB}$. However, in the low frequency region up to $0.1 \mathrm{~Hz}$, the control effect is not apparent. This is because the disturbance dynamics gain of the trolley wire is reduced.

Figures 10 ( $a$ ) and ( $b$ ) show the frequency characteristics of contact force $(f(t))$ and control force $\left(F_{p}(t)\right)$ with respect to the lift disturbance $\left(f_{\text {lift }}(t)\right)$. At low frequencies up to $0.1 \mathrm{~Hz}$, the phase of active control is advanced and exhibits a controlling effect. Consequently, it is found that the lift disturbance is

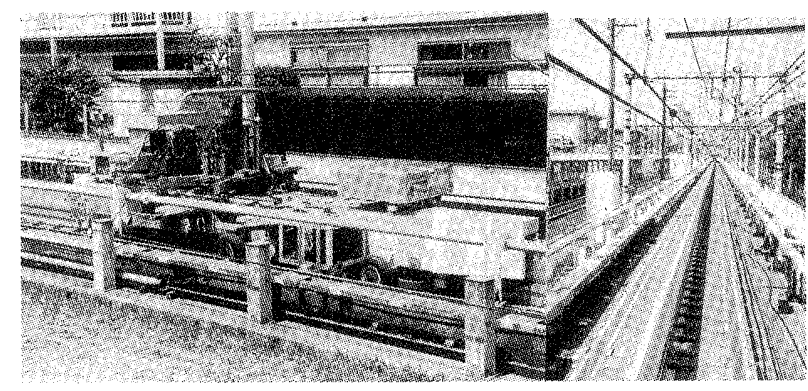

Fig. 11 Photograph of a full-size prototype current collector on the running vehicle

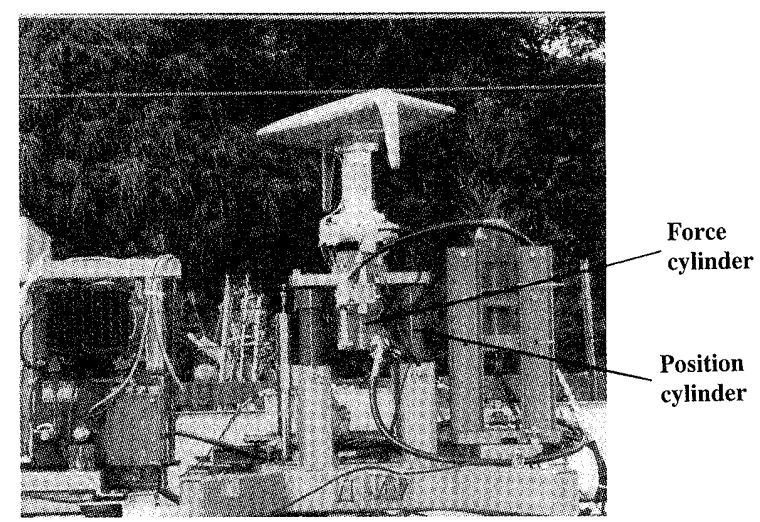

Fig. 12 Photograph of a full-size prototype current collector

effectively suppressed with respect to steady lift below $0.1 \mathrm{~Hz}$. Since the current collector is firmly supported by the supporting insulator, the force cylinder and other parts, it has an inherent frequency of oscillation of over $20 \mathrm{~Hz}$. The lift disturbance principally consists of a steady lift in the low-frequency region. Thus, it seems to be sufficient to have good suppression performance in the low-frequency region. As mentioned above, it was found to be possible to suppress the disturbance of, for example, trolley wire irregularities and steady lift. The contact force is clearly set to the desired value.

\section{Experimental Results and Study}

\subsection{Experimental results using running vehicle of the Railway Technical Research Institute}

Figures 11, 12 and 13 show the mounting structure and the setup for a test run of experimental equipment including a full-size prototype. As shown in Fig. 13, the $200 \mathrm{~kg}$ prototype was mounted at the center of the vehicle, and behind it were the hydraulic source and an electrical generator for supplying it with electricity. Also, to detect the speed of the vehicle, a noncontacting Doppler speedmeter was attached to the side of the vehicle ${ }^{(17)}$.

Figure 14 shows the trolley wire height vibration, the lift and the traveling speed of the passive system 


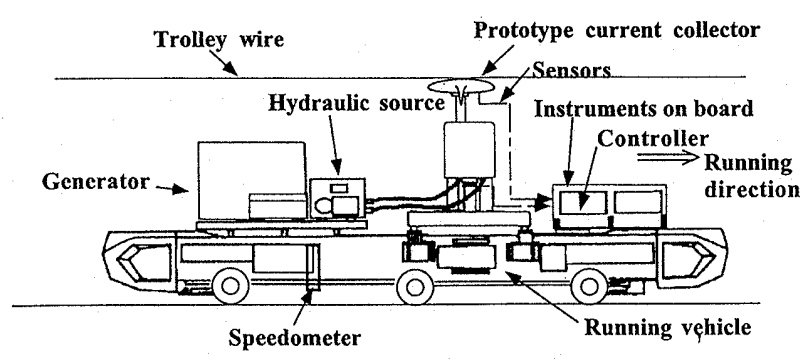

Fig. 13 Configuration of a full-size prototype current collector on the running vehicle
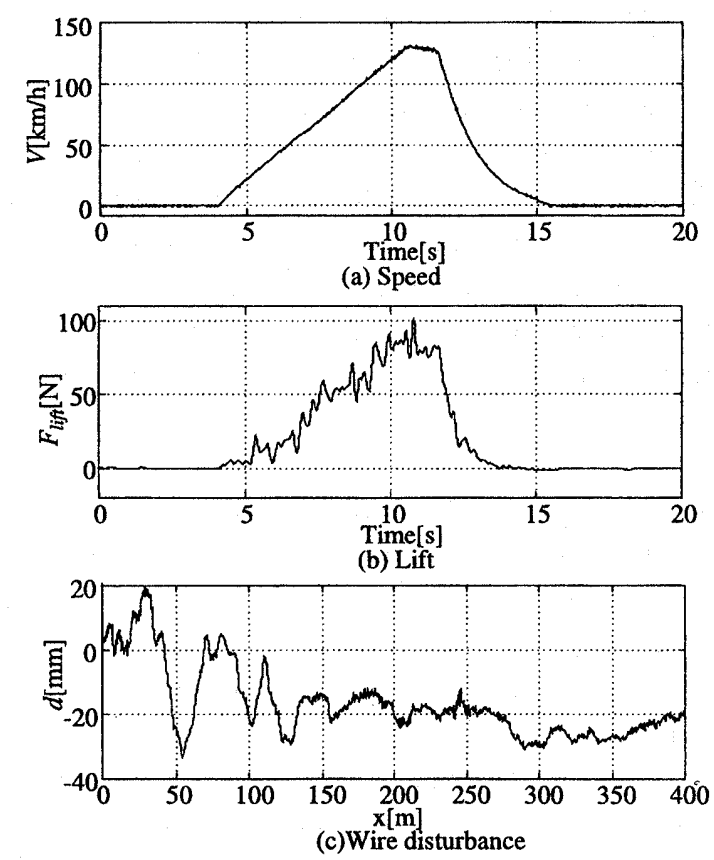

Fig. 14 Experimental results acting on the prototype current collector shown in Fig. 11. As shown in Fig. 14 (c), the height of the trolley wire varied between +20 to $-30 \mathrm{~mm}$ about the average height of $2120 \mathrm{~mm}$ above the railroad surface. Also, Figs. 14(a) and (b) show the lift waveform acting on the prototype current collector and the speed waveform at a maximum speed of 130 $\mathrm{km} / \mathrm{h}$. From this result, it is shown that, in the coasting region from 7 to 8.5 seconds after the start of the run, when the vehicle was moving at the maximum speed of $130 \mathrm{~km} / \mathrm{h}$ (acceleration $0.35 \mathrm{~g}$, deceleration $1.0 \mathrm{~g}$ ), an average lift of about $90 \mathrm{~N}$ (time waveform, cut above $2 \mathrm{~Hz}$ by a low-pass filter) acted on the prototype current collector in the direction of raising the trolley wire. Because the maximum speed of the test run with the active system was set at $112 \mathrm{~km} / \mathrm{h}$, the disturbance of the steady lift was about $68 \mathrm{~N}$.

Figure 15 shows the results of active control with lift compensation and without lift compensation. In active control without lift compensation, shown on the left side of Fig. 15, the contact force is controlled without considering the lift, so that when traveling at a coasting speed of $112 \mathrm{~km} / \mathrm{h}$, an average lift of $68 \mathrm{~N}$ acts upwards, and the maximum contact force $(f(t))$ becomes $205 \mathrm{~N}(+107 \mathrm{~N})$, which is about twice the contact force before the run. The average observed value of the load cell at this time was the desired value of $98 \mathrm{~N}$. Also, the contact force between the trolley wire and the contact strip was the sum of the observed value of the load cell $(98 \pm 22 \mathrm{~N})$, the average

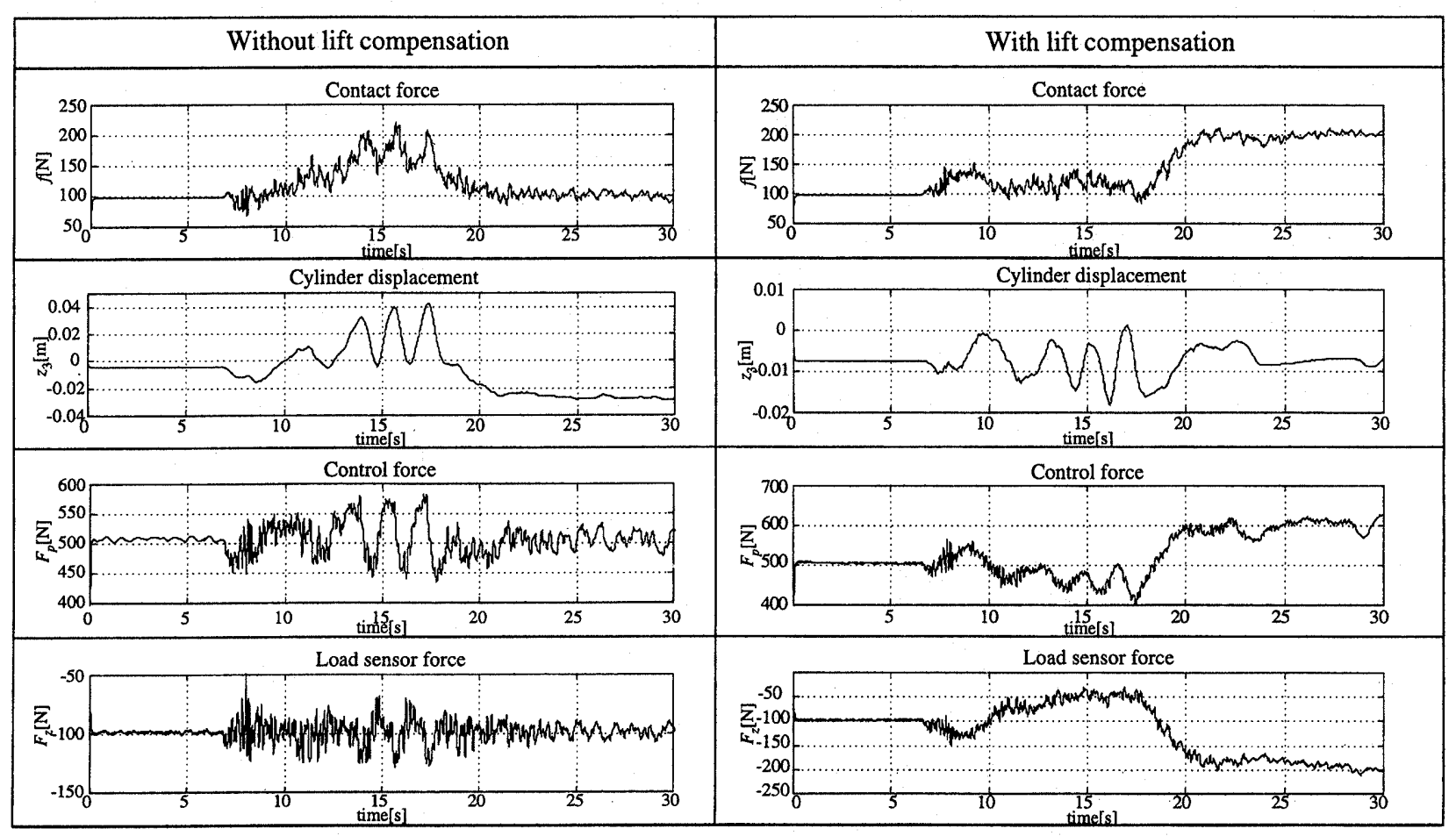

Fig. 15 Experimental results using running vehicle of the R.T.R.I. 
lift of the prototype current collector $(68 \mathrm{~N})$, and the variable force component of the contact strip, and was in the range of 127 to $205 \mathrm{~N}$. Furthermore, the displacement of the force cylinder corresponded to the vertical oscillation of the trolley wire, which was a 1 $\mathrm{Hz}, 42 \mathrm{~mm} \mathrm{p}^{-} \mathrm{p}$ sinusoidal wave. The hydraulic force at this time was superimposed with a $147 \mathrm{~N} \mathrm{p}-\mathrm{p}$ fluctuating component on $510 \mathrm{~N}$, which included the inherent stationary weight component $(412 \mathrm{~N})$ and the initial force component $(98 \mathrm{~N})$.

In the active control of lift compensation shown on the right side of Fig. 15, the contact force is controlled while considering lift, so that when traveling in the coasting speed range of $112 \mathrm{~km} / \mathrm{h}$, the maximum contact force was about $130 \mathrm{~N}(+32 \mathrm{~N})$, which is about 1.3 times the contact force before the run. The average observed value of the load cell at this time was $50 \mathrm{~N}$, which was smaller than the desired value $(98 \mathrm{~N})$. Also, the contact force was the sum of the observed value of the load cell $(50 \mathrm{~N})$, the average lift of the wing-shaped current collector (68 $\mathrm{N}$ ), the variable force component of the contact strip and the half-peak amplitude $14 \mathrm{~N}$, and was in the range of 112 to $130 \mathrm{~N}$. Furthermore, the displacement of the force cylinder corresponded the vertical oscillation of the trolley wire, which was a $1 \mathrm{~Hz}, 10 \mathrm{~mm} \mathrm{p}^{-} \mathrm{p}$. The hydraulic force was superimposed with a fluctuating component of about $60 \mathrm{~N} \mathrm{o}$-p on $510 \mathrm{~N}$, including the inherent stationary weight component $(412 \mathrm{~N})$ and the initial force component $(98 \mathrm{~N})$.

Figure 16 shows the time waveforms of the pushup wire displacement at the center of trolley wire spans. The dotted line shows the case without lift compensation, where the amount of push-up was about $75 \mathrm{~mm}$ at the center of the span, and oscillation with a frequency of about $1.0 \mathrm{~Hz}$ occurred after the vehicle had passed by. Also, the solid line shows the case of lift compensation, where the amount of lift was about $50 \mathrm{~mm}$ at the center of the span. The amount of push up of the trolley wire is smaller in the latter case. These results demonstrate the effect of active control with lift compensation, and show that the wire push-up variation is reduced to about twothirds $(68 \%)$.

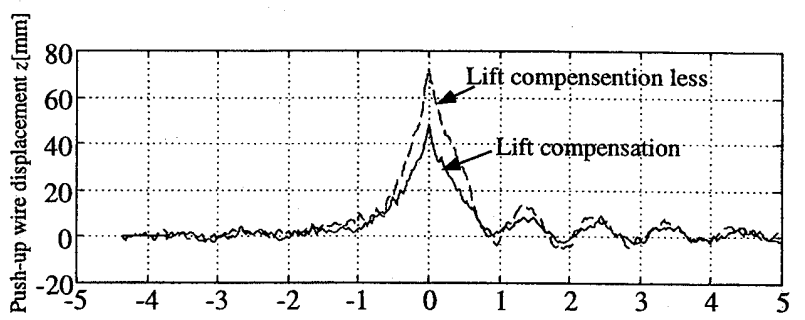

Fig. 16 Push-up wire displacement for full-size prototype current collector using active controls
As stated above, when coasting at a speed of 112 $\mathrm{km} / \mathrm{h}$, we have confirmed that it is possible to reduce the wire push-up variation to $2 / 3$ and the variable component of the maximum contact force to $1 / 3$ with a steady lift of about $68 \mathrm{~N}$. However, during rapid deceleration 20 seconds after the start of the run and when the speed is low before stopping (in the frequency band lower than $0.1 \mathrm{~Hz}$ ), it has been found that lift compensation is affected by the force cylinder where the displacement of about $30 \mathrm{~mm}$ of the trolley wire irregularity is judged to be.

\section{2 Simulation study of contact force control system with additional trolley wire height control}

The following study was performed using a position cylinder supporting the force cylinder, as shown in Fig. 1 (b), with the aim of tracking the fluctuation of the trolley wire height. The position cylinder operated independently of the force cylinder, and its position was controlled by proportional and integral control. With this structure, the force cylinder controls the steady lift (low-frequency component) and the displacement disturbance of trolley wire irregularities (high-frequency component). The position cylinder performs cooperative control to control the height to the displacement disturbance of the trolley wire irregularities (low-frequency component). Figure 17 shows a block diagram of this cooperative control system. The top part of the figure is the force control system, and the bottom part of the figure is the height control system. The proportional integral compensation system and the dynamics of the position cylinder shown in Fig. 12 were experimentally determined as described above. They were fitted to a second-order system $\left(\zeta_{0}=0.7, \omega_{0}=2 \mathrm{~Hz}\right)$. Accordingly, the observed quantity $z_{3}(t)$ used in the contact force control system of the force cylinder is changed to $z_{3}(t)-z_{4}(t)$, and the detected values are expressed by

$$
\boldsymbol{y}(t)=\left[\begin{array}{llll}
f_{z}(t) & z_{3}(t)-z_{4}(t) & \ddot{z}_{2}(t) & \ddot{z}_{3}(t)
\end{array}\right]^{T}
$$

$z_{4}(t)$ is absolute displacement of the position cylinder.

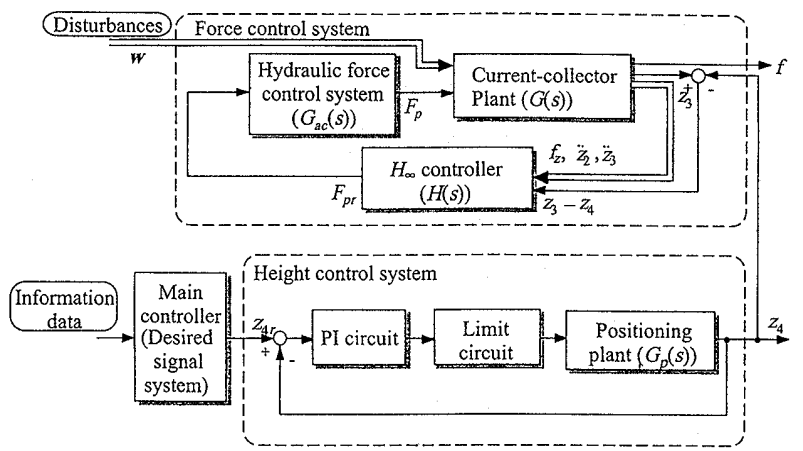

Fig. 17 Block diagram of total control system with a force control system and height control system 


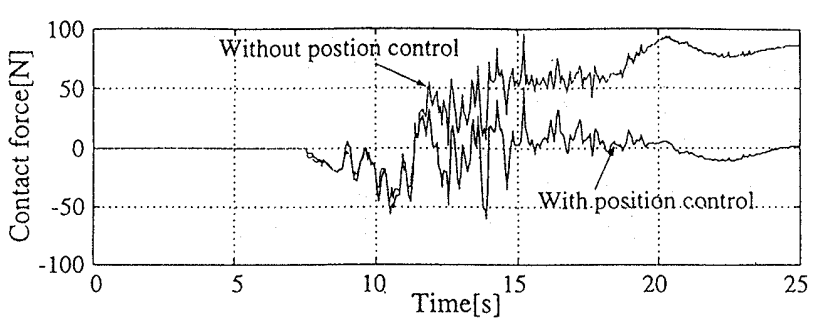

(a) Contact force $(\boldsymbol{f})$

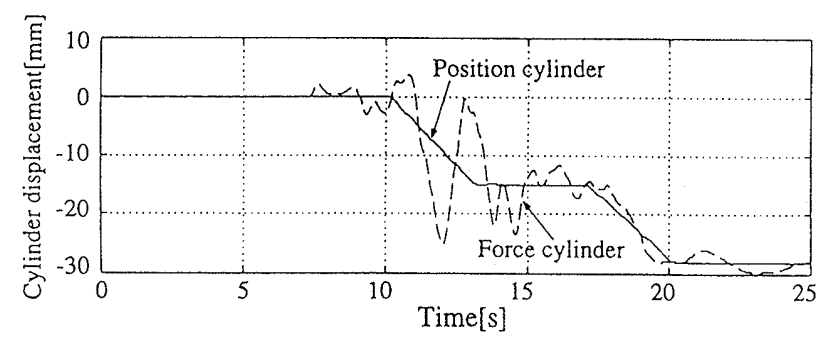

(b) Cylinder displacemnt $\left(z_{3}, z_{4}\right)$

Fig. 18 Time response of contact force

Figure 18 shows the simulated results of adding trolley wire height control with a position cylinder, as described above. In this figure, the dotted line shows the case without trolley wire height control, and the solid line shows the case with trolley wire height control. These results show that the position cylinder tracks the desired position and suppresses the displacement disturbance of the trolley wire irregularities that cannot be tracked by the force cylinder. Also, it is clear that by separating periodic components from the displacement of the force cylinder, the position cylinder is able to track the high-frequency region of the displacement disturbance of the trolley wire irregularity. Accordingly, it offers the prospect of being able to set the fluctuation of the contact force close to the desired value, according to the low-frequency region of the trolley wire irregularity which is produced when the train is stationary or traveling at low speed.

\section{Conclusions}

In a low-noise current collector, it is important to have a trolley wire tracking performance similar to those hitherto achieved. We have proposed a contact force control method for this purpose, and we have designed and simulated a controller using this method. Also, from running tests experiments performed at $\mathrm{R}$. T.R.I. using a full-size prototype, the following trolley wire tracking performance has been obtained.

(1) We proposed a contact force $H_{\infty}$ control method whereby four detectors are provided on the ground voltage part below the observable supporting insulator.

(2) This controller classifies disturbance accord- ing to frequency into a low-frequency region and a high-frequency region. We have confirmed in simulations that steady lift up to $0.1 \mathrm{~Hz}$ can be reduced, and that has good tracking performance due to trolley wire irregularities between 0.2 and $6 \mathrm{~Hz}$. However, the control performance tends to decrease in the highfrequency region.

(3) In test runs (coasting speed $112 \mathrm{~km} / \mathrm{h}$ ) of the current-collector running vehicle for trolley wire tracking, we were able to suppress the variation of the maximum contact force (when the lift was $68 \mathrm{~N}$ ) to 30 $\mathrm{N}$ (about one-third), and we confirmed the tracking performance of trolley wire irregularities to be $50 \mathrm{~mm}$ (about two-thirds) in wire push-up variation.

(4) By performing height control, using a position cylinder, with respect to trolley wire height variation, our simulations offer the prospect of reducing the low-frequency component of trolley wire fluctuation.

\section{Acknowledgements}

The authors appreciate the efforts of Dr. K. Manabe, Director, Mr. M. Ikeda, Chief Researcher of Railway Technical Research Institute, and $\mathrm{co}^{-}$ researchers of Japan Railway companies, in the coresearch to promote this development.

\section{References}

(1) Ohyama, T., RTRI Report, (in Japanese), Vol. 8, No. 6, (1994), p. 1.

(2) Yoshikawa, T., Machine Research, (in Japanese), Vol. 46, No. 10(1994), p. 1020.

(3) Moritoh, Y. and Zenda, Y., RTRI report, (in Japanese), Vol. 8, No. 6(1994), p. 7.

(4) Manabe, K., JREA, (in Japanese), Vol. 38, No. $8(1995)$, p. 10.

(5) Morikawa, T. and Ikeda, M., RTRI report, (in Japanese), Vol. 8, No. 6(1994), p. 19.

(6) Iida, A., Takano, T., Makino, T. and Kobayashi, K., INTER-NOISE 94,(1994), p. 163.

(7) Makino, T., Kakehi, Y., Iida, A., Terada, K. and Hattori, M., Trans. Jpn. Soc. Mech. Eng., (in Japanese), Vol. 63, No. 612, C (1997), p. 2679.

(8) Yoshida, K. and Shimogo, T., Trans. Jpn. Soc. Mech. Eng., (in Japanese), Vol.54, No. 504, C (1988), p. 1821.

(9) Yoshida, K., Trans. Jpn. Soc. Mech. Eng., (in Japanese), Vol. 48, No. 427, C(1982), p. 358.

(10) Thompson, A. and Davis, B., Vehicle System Dynamics, Vol. 19(1990), p. 131.

(11) Galeotti, G., Galanti, M. and Magrini, S., Proc. Instn. Mech. Engrs. Part F, Vol. 207 (1993), p. 37.

(12) Kobayashi, K., Makino, T., Kakehi, Y. and Iida, A., J-RAIL '95, (in Japanese), No. 81 (1995), p. 287.

(13) Fujii, S. and Shibata, H., Proc. of the Japan National Cong. for Applied Mechanics, (1957), p. 375 .

(14) Noritugu, T., Trans. Soc. Ins. Control. Eng., (in 
Japanese), Vol. 29, No. 1 (1993), p. 86.

(15) Makino, T., Yoshida, K. and Seto, S., Trans. Japan. Soc. Ins. Control. Eng., (in Japanese), Vol. 63, No. 611, C (1997), p. 2287.

(16) Mita, T., $H_{\infty}$ Control; Syoukoudou, (in Japanese), (1994).

(17) Ikeda, M. and Manabe, K., RTRI report, (in
Japanese), Vol. 6, No. 9(1992), p. 13.

(18) Doyle, J., Glover, K., Khargonekar, P.P. and Francis, B.A., IEEE Trans. on Automatic Control, Vol. AC-34, No. 8(1989), p. 831.

(19) Sampei, M., Mita, T. and Nakamichi, M., System and Control Letters, Vol. 14 (1990), p. 13. 